\title{
Under the Processing of Network Data Images, the Position Recognition Technology of Mechanical Parts Based on "Mathematical Modeling" is Studied
}

\author{
Jiaqi Zhao ${ }^{1}$ \\ ${ }^{1}$ Jilin Railway Technology College,Jilin China, 132200
}

\begin{abstract}
With the development of the times and the progress of society, science and technology has been developing more and more rapidly. In this regard, modern society has been composed of science and technology. Compared with the original steam engine, Jenny textile machine and other industrial era starters, now the technology has entered the era of large machines. As a result, the processing of mechanical parts is becoming more and more complex than before. Therefore, the purpose of this paper is to use the network data image processing based on mathematical modeling of mechanical parts processing location recognition technology. After consulting the relevant literature on mechanical parts processing and location recognition technology, this paper goes to the factory to observe the mechanical working principle and mechanical working process, and then uses the two-dimensional spectral peak search algorithm to design the position recognition technology in the process of mechanical parts processing to process mechanical parts based on mathematical modeling. The experimental results show that the two-dimensional spectral peak search algorithm can make the system better point out the relevant problems in the process of mechanical parts processing and solve them intelligently, and if there are problems that cannot be solved, it will be fed back to the system informer in time to check. Finally, the accuracy and correctness of mechanical parts processing are improved, and some improvements are put forward for mechanical parts processing, which is of great help to mechanical parts processing.
\end{abstract}

\section{Introduction}

Because the arrival of the industrial age has been a long time, modern life is composed of science and technology, and due to the vigorous development of modern computer technology, we have entered an era of rapid development [1]. In order to have more say in the future, we should vigorously develop network technology and computer technology. Because only in this way can we make use of more advanced modern science and technology to make some more perfect industrial products to serve our life [2]. Therefore, in modern life, mechanical intelligence has gradually replaced artificial manufacturing. However, there are many problems in the processing of mechanical parts, which are prone to defects [3]. So the purpose of this paper is to use the network data image processing to find the position recognition technology in the machining process of mechanical parts based on mathematical modeling.

The history of machinery manufacturing in China is only a few decades, which is not as good as that in Europe and America. However, due to China's subsequent efforts to promote the development of manufacturing industry, China's manufacturing industry is now basically complete and self-sufficient [4]. But many high-end technologies can't be manufactured for the time being, such as lithography machine, bomber engine and so on. But these technologies are indispensable in modern life [5]. For example, chips made by lithography machines are widely used in computers and mobile phones, and chips are the brains of computers and mobile phones, so we have to import high-precision products from other countries. The engine, not to mention, is one of the cores determining whether China's aircraft can develop into a world-class bomber [6]. However, it is not feasible to rely on importing high-end processing parts from other countries. For example, the trade war last year and Huawei's chip fault show that as long as China does not manufacture its own high-end products, it will always be threatened by other countries. Therefore, we should vigorously develop our own machinery manufacturing industry and increase support for the development of high-end technology. However, the development of these technologies is very difficult, and it will take generations of accumulation to realize it, so as to gradually surpass it [7]. Therefore, we should make greater efforts to produce better and more sophisticated processing parts, to keep up with the world's first-class products, to break the shackles of technology and strive for China's position in the world history. Therefore, we need to use the network data image to analyze the machining process of mechanical parts based 
on mathematical modeling in an all-round way, and then use the position recognition technology to study this, so as to produce better and more sophisticated mechanical parts [8].

Mechanical design and mechanical manufacturing are two aspects of content, but they are an important part of mechanical engineering. Mechanical design is the first step of mechanical production. Only design first can decide the next step. So design is the most important thing in the whole process. Only after a process is designed, can we start construction, and then carry out mechanical manufacturing, so as to produce the goods we need [9]. But a good design can't do without corresponding production ability, so the two complement each other and should go hand in hand for common development. So we can't start with hardware, so we start with software. Therefore, based on the two-dimensional spectral peak search algorithm, this paper proposes solutions to some problems in mechanical design and manufacturing, and studies the position recognition technology in the machining process of mechanical parts based on mathematical modeling under the network data image processing, so as to facilitate the better completion of mechanical manufacturing [10].

\section{Algorithms and modeling}

Array flow vector module $\alpha(\theta, \varphi)=\exp \left\{-\mathrm{j} \frac{2 \pi}{\lambda}(x \cos \theta \cos \varphi+y \sin \theta \cos \varphi)\right\}$

The purpose of this module is to calculate (1), select a set of axons and elevated angles, and obtain an $8 \times 1$ matrix, corresponding to 8 arrays. Remember the new matrix $\mathrm{X}$ to make it satisfying(2); And make the new matrix $Y$ satisfied(3), available

$$
\begin{aligned}
& \mathrm{X}=360(\mathrm{x} \cos \theta \cos \varphi+\mathrm{y} \sin \theta \cos \varphi) \\
& \mathrm{Y}=\mathrm{X} / \lambda \\
& \text { Then there is } \\
& \alpha(\theta, \varphi)=\cos \mathrm{Y}-\mathrm{j} \sin \mathrm{Y}
\end{aligned}
$$

In order to reduce the amount of computation, preprocessing is required to complete the conversion from complex domain to real domain without changing the final result. Therefore, for the $1 \mathrm{st}, 2 \mathrm{nd}, 3 \mathrm{rd}$, and 4 th formations, there is

$$
\alpha(\theta, \varphi)=\cos Y
$$

And for the 5th, 6th, 7th, 8th array, there is

$$
\alpha(\theta, \varphi)=\sin Y
$$

Establish an early warning system model

By analyzing the relationship between the parameters in the early warning system indicator system, two or two comparisons are made between the indicators at the same level, the model matrix is constructed, the characteristic value of the model matrix and its characteristic vector are calculated, and a test is carried out after the weight vector is derived.

Build two or two comparative indicators to judge matrix R:

$$
\begin{aligned}
& R=\left(r_{i j}\right)_{m * n} \\
& i=1,2, \ldots, m \\
& j=1,2, \ldots, n
\end{aligned}
$$

Make the things in $\mathrm{R}$ a planning process:

$$
\begin{aligned}
& \mathrm{r}_{\mathrm{ij}}^{\prime \prime}=\mathrm{r}_{\mathrm{ij}} / \sum_{\mathrm{i}=1}^{\mathrm{n}} \mathrm{r}_{\mathrm{ij}} \\
& \mathrm{r}_{\mathrm{ij}}^{\prime \prime}=\sum_{\mathrm{i}=1}^{\mathrm{m}} \mathrm{r}_{\mathrm{ij}} \\
& \mathrm{W}_{\mathrm{IJ}}=\mathrm{r}_{\mathrm{ij}} / \sum_{\mathrm{i}=1}^{\mathrm{n}} \mathrm{r}_{\mathrm{ij}}^{\prime \prime} \\
& \mathrm{A}_{\mathrm{w}}=\lambda_{\max } \mathrm{W} \quad(13) \\
& \mathrm{CI}=\frac{\lambda_{\max }-\mathrm{n}}{\mathrm{n}-1} \quad \mathrm{CR}=\frac{\mathrm{CI}}{\mathrm{RI}}
\end{aligned}
$$

In the process of building the model above, RI is a random consistency indicator, if the CR-1t; 0.1 , then the consistency test passes, otherwise you will continue to correct the $\mathrm{R}$ of the indicator judgment, the above model fusion and, headphone indicator evaluation weight, obtain the comprehensive evaluation score, and determine the corresponding seismic rating formula as follows:

$$
\mathrm{E}=\mathrm{F} * \mathrm{~W} * \mathrm{~V}
$$

\section{Experiment}

\subsection{Selection of experimental sites}

Because the requirements of the experiment were not very high, we borrowed from the school a laboratory that could be used for leisure time for the experiment. The laboratory has hydraulic presses, flattening synthesies, rolling workshops, milling cutters and other mechanical tools, as well as displays, counters, computers, adapters and other digital tools, these are all the equipment we need for our experiments. Subsequently, we use the synthesum to form each tool into a simple workshop, with rolling cutting, flat transport and other functions, and then we made the system and the original system are processed and made separately, and finally came up with a series of finished products, and then the measurement data to compare the parameters.

\subsection{Extraction of experimental data}

We have carried out many experiments and derived dozens of sets of data from them, and then extracted them for comprehensive comparative analysis, using linear regression coupling to explore the validity of the data we obtain, there will be several sets of error data troubleshooting, and then linear analysis, and finally come up with a better set of data, they are analyzed and compared to the final is the experimental results we need.

\section{Evaluation results}

\subsection{The results of the experiment are compared}

Table 1 parameter comparison

\begin{tabular}{|c|c|c|}
\hline & $\begin{array}{c}\text { Original } \\
\text { machinery } \\
\text { manufacturin } \\
\mathrm{g}\end{array}$ & $\begin{array}{c}\text { Machinery } \\
\text { manufacturin } \\
\mathrm{g}\end{array}$ \\
\hline Rotation rate m/s & 14 & 16 \\
\hline $\begin{array}{c}\text { Turn the speed } \\
\mathrm{rad} / \mathrm{s}\end{array}$ & 3600 & 4200 \\
\hline
\end{tabular}




\begin{tabular}{|c|c|c|}
\hline Flattening rate $\mathrm{m} / \mathrm{s}$ & 8 & 11 \\
\hline $\begin{array}{c}\text { Flattening rate } \\
\mathrm{rad} / \mathrm{s}\end{array}$ & 2100 & 2600 \\
\hline Relative efficiency & $26 \%$ & $35 \%$ \\
\hline
\end{tabular}

Based on a comparative analysis of Table 1 data, we find that there is a greater improvement in mechanical manufacturing in all aspects than in the original mechanical manufacturing. Overall, the increase is around $10 \%$. Of course, this is only a few simple tests to get the paper parameters, so we also carried out the actual production test, the results are shown in the Figure below.

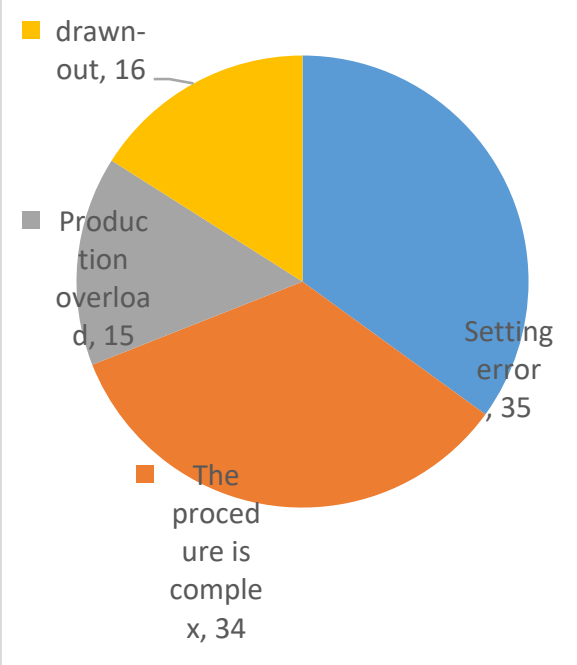

Figure 1 The cause of the original machinery manufacturing production error

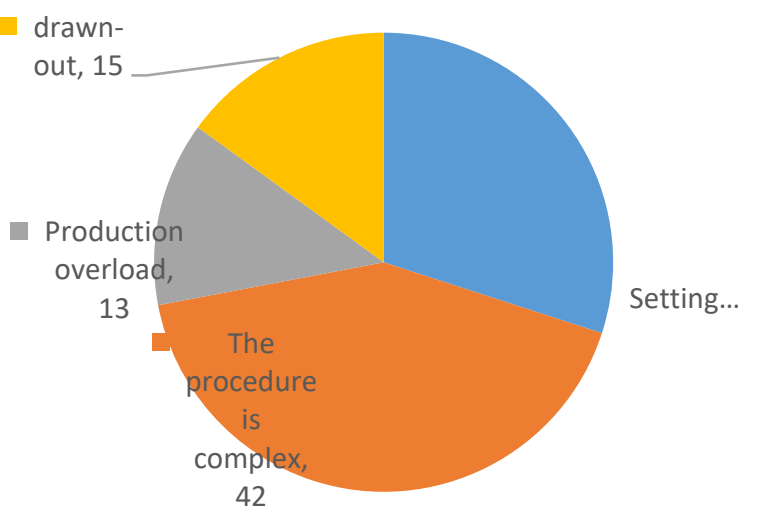

Figure 2 The cause of the manufacturing error in machinery

As the chart information shows, based on the corrected data, the main errors are still due to procedural complexity and set-up errors, so we will step up our efforts to change the settings for both.

\subsection{Mechanical design}

Mechanical design is based on our needs to work on mechanical work parts, each part, the mode of motion, as well as the transfer of energy, as well as the materials of each component and shape size and other parts of the conception and then to carry out the overall grasp, into a specific flow chart, become the basis for the work of our manufacturing machinery. Good mechanical design is to make the best mechanical use of the minimum cost under various qualification factors, that is, the traditional sense of optimization design.

Of course, mechanical design is also divided into two categories, one is a specific design, the other is a general design. A particular design requires a comprehensive consideration of many requirements. For example, the mechanical environment acidity, temperature, radioactive intensity and other factors, and these need to be considered in detail to select materials. Although the design is the most suitable and best machine, but in general, most of us still design general design, that is, the design in all cases, the performance of the better machinery. Although the performance is not perfect, it works in most environments.

Mechanical design can also be divided into three types of design, namely, new design, inherited design and deformation design. The new design is the application of existing tested science and technology to design a new type of machinery that has never been seen before. Inheritance technology is the use of existing experience and machinery, to update it, improve its performance and reduce its cost. The deformation design is to make some modifications to the existing machinery and become a product suitable for all kinds of situations.

The design phase is also divided into many process stages. Because mechanical design is dynamic, it is subject to changes and adjustments during the production process to meet real-world design conditions and make modifications to achieve objectives. First of all, for the machine we need to design to do a full and comprehensive investigation, and analysis, and then learned that the machine should have the function and general production costs, so that we have a whole control, suitable for future design to add a variety of restrictions, and on this basis on the design task requirements for a reasonable control; the second stage is scenario design. We can set a variety of production scenarios according to the different working principles of the machinery we manufacture, for example, the machine tool can be divided into flat and rolling, cutting and grinding, so we will set up a variety of schemes at this time. However, in these scenarios, you can apply only a few of the actual, and then from these, according to the qualification criteria, to select one or several of the best ones for design, and at this stage should be bold examples, careful proof. In the next phase, we're going to do the technical design. To mechanically draw a variety of component drawings, this is a complex and huge project, to make the various parts unified, and calculate the run-in between, select the best parts to use. Then, in order to make the size of the part, we need to know how the machine is moving, the load on the parts and machinery, as well as the working ability of the parts, as well as the assembly sketches of the parts and the overall assembly sketches, and perform assembly checks to determine the use of the parts. Finally, it is to make the finished product, to its various parts of the environment of the experiment, to get the parameters we need to see if it meets expectations. If the target is not achieved, identify the cause and improve it to get the target machinery it deserves. 


\subsection{Machinery manufacturing}

The production process of machinery manufacturing is generally the whole process of turning raw materials into finished products. Generally, includes pre-production technical preparation, this time is the design of the production plan. The blanks are then forged and parts processed, and the finished product is supplied and sold after the product is equipped.

The production type of machinery manufacturing is generally divided into three categories, single-piece production, mass production, mass production. Singlepiece production is generally suitable for delicate and expensive small pieces. And this production may also be designed for large machinery to be tested to decide whether to mass produce and then mass production. Mass production can be divided into large, medium and small three levels, generally according to the number of productions to the level. Finally, mass production, that is, the number of manufacturing is particularly large. Generally, is a large supply and demand and few varieties, low production prices and a wide range of applications of a class of products.

This is followed by product design. The mechanical design can be referred to. After that is process design, parts processing, inspection. Process design must first meet the basic tasks of the product: good quality, high output, low energy consumption, and drawings should be perfect, audit needs to pass, there is a reasonable and clear processing program, complete process procedures and complete process equipment. The parts are then machined, and the procedure includes the use of blanks and excess blanks for precision forging. In general, there are fitters, car mechanics, cutters, boring workers, $\mathrm{CNC}$ machine tools and other processing, and then there are positive, quenching and other fire treatment processes and electric sparks, laser processing and other special processing processes. Of course, these are arranged according to the material, size and nature of the part.

\section{Conclusion}

In summary, we can learn from this experiment that the use of two-dimensional spectral peak search algorithm can better for the network data image processing and mathematical modeling of mechanical parts processing processing location recognition technology for development and design. But there may be some flaws in it, so purely using existing technology doesn't completely solve all the problems we're experiencing. Therefore, what we are doing now is only an early attempt, and we believe that there will be better solutions in the future.

\section{Resources}

1. Ueda H, Yamada T, Miwa T, et al. Development of a Data Sharing System for Japan Volcanological Data Network[J]. Journal of Disaster Research, 2019, 14(4):571-579.

2. Tang Y, Xiong J J, Luo Y, et al. How Do the Global Stock Markets Influence One Another? Evidence from Finance Big Data and Granger Causality Directed Network[J]. International Journal of Electronic Commerce, 2019, 23(1):85-109.

3. Hollstein B, Tpfer T, Pfeffer J. Collecting egocentric network data with visual tools: A comparative study[J]. Network Science, 2020, 8(2):223-250.

4. Malyar V. Mathematical modeling start-up and steady state modes of asynchronous motors operation with capacitive compensation of reactive power[J]. Przeglad Elektrotechniczny, 2020, 1(11):111-116.

5. Yang Z, Shao D, Zhou G . Investigation of Gramine Solubility and Mathematical Modeling in Various Pure Solvents and the Co-Solvent Mixture of (Ethanol + Ethyl Acetate) at (273.15-313.15) K[J]. Journal of Chemical And Engineering Data, 2020, 65(4):14881496.

6. Kong L, Yang X, Hou Z, et al. Mathematical Modeling of Drying Kinetics for Pulp Sheet Based on Fick's Second Law of Diffusion[J]. Palpu Chongi Gisul/Journal of Korea Technical Association of the Pulp and Paper Industry, 2020, 52(2):23-31.

7. Yuanyuan, Guo, Youchao, et al. Reliability assessment for multi-source data of mechanical parts of civil aircraft based on the model[J]. Journal of Mechanical Science and Technology, 2019, 33(7):3205-3211.

8. $\mathrm{Xu} \mathrm{Z}$, Wang $\mathrm{Y}, \mathrm{Wu} \mathrm{D}$, et al. The process and performance comparison of polyamide 12 manufactured by multi jet fusion and selective laser sintering[J]. Journal of Manufacturing Processes, 2019, 47(Nov.):419-426.

9. Tanabe E H, Silva R M , Daliomar L.Oliveira Júnior, et al. Recovery of valuable metals from waste cables by employing mechanical processing followed by spouted bed elutriation[J]. Particuology, 2019, 45(004):74-80.

10. Lee J W , Choi Y H , Kim Y B , et al. A Study on Position Estimation and Path Planning Technology of Autonomous Underwater Vehicle (AUV)[J]. Journal of Power System Engineering, 2020, 24(3):60-66. 